\title{
Understanding the infant feeding decisions of low-income Irish women
}

\author{
Emily Shortt ${ }^{1}$, Catherine McGorrian ${ }^{1}$, Orla Doyle ${ }^{2}$, Jean Kilroe ${ }^{3}$ and Cecily Kelleher ${ }^{1}$ \\ ${ }^{1}$ School of Public Health, Physiotherapy and Population Science and ${ }^{2}$ Geary Institute, University College Dublin, Belfield, \\ Dublin 4, Republic of Ireland and ${ }^{3}$ Riche Project FP 7, Dublin City University, Dublin 9, Republic of Ireland
}

Breast-feeding rates are lower in Ireland than other westernised countries and are particularly low among low-income families. The purpose of this qualitative study was to explore the infant feeding decisions of low-income Irish women and to identify perceived barriers and facilitators to breast-feeding initiation and continuation. Four focus groups and 13 semi-structured interviews were carried out with a total of 33 low-income mothers recruited from a variety of urban and rural settings.

Seven dominant themes exploring infant feeding decisions were identified from an analysis of the interview transcripts: (1) prior knowledge about breast-feeding; (2) embarrassment about breast-feeding in the public and private sphere; (3) balancing the health and welfare needs of the mother and baby; (4) convenience and freedom; (5) self-determination about feeding choice; (6) early difficulties with breast-feeding and the need for experienced support and (7) health professional support.

The women perceived that breast-feeding is not the 'normal' thing to do in their social context. They also perceived that breast-feeding in public is not well accepted, especially if it involves bodily exposure. Breast-feeding mothers perceived a social pressure to hide from public gaze, which restricts other women's knowledge about breast-feeding. Many of the women automatically followed the infant feeding traditions of their family, while those who changed to breast-feeding often lacked experienced support to help them overcome difficulties. An analysis of the women's dialogues suggested that the low acceptability of breast-feeding in Irish society derives from traditional, cultural associations of the breast with sexual acts and immorality, which makes breast-feeding seem unnatural.

These findings suggest that public health promotion strategies should focus on changing societal attitudes towards breast-feeding. In addition, written materials alone do not adequately address the informational needs of low-income women; ongoing practical support from health professionals and experienced breast-feeders is needed during the antenatal and postnatal period to help women cope with breastfeeding difficulties. 